\title{
Halo histories versus galaxy properties at $z=0$ II: large-scale galactic conformity
}

\author{
Jeremy L. Tinker, ${ }^{1 \star}$ ChangHoon Hahn, ${ }^{1}$ Yao-Yuan Mao, ${ }^{2}$ Andrew R. Wetzel ${ }^{3,4,5} \dagger$ \\ and Charlie Conroy ${ }^{6}$ \\ ${ }^{1}$ Center for Cosmology and Particle Physics, Department of Physics, New York University, New York, NY 10003, USA \\ ${ }^{2}$ Department of Physics and Astronomy and Pittsburgh Particle Physics, Astrophysics, and Cosmology Center (PITT PACC), University of Pittsburgh, PA \\ 15260, USA \\ ${ }^{3}$ Carnegie Observatories, Pasadena, CA 91101, USA \\ ${ }^{4}$ TAPIR, California Institute of Technology, Pasadena, CA 91125, USA \\ ${ }^{5}$ Department of Physics, University of California, Davis, CA 95616, USA \\ ${ }^{6}$ Department of Astronomy, Harvard University, Cambridge, MA 02138, USA
}

Accepted 2018 March 9. Received 2018 February 27; in original form 2017 March 22

\begin{abstract}
Using group catalogues from the Sloan Digital Sky Survey (SDSS) Data Release 7, we measure galactic conformity in the local universe. We measure the quenched fraction of neighbour galaxies around isolated primary galaxies, dividing the isolated sample into star-forming and quiescent objects. We restrict our measurements to scales $>1 \mathrm{Mpc}$ to probe the correlations between halo formation histories. Over the stellar mass range $10^{9.7} \leq M_{*} / \mathrm{M}_{\odot} \leq 10^{10.9}$, we find minimal evidence for conformity. We further compare these data to predictions of the halo age-matching model, in which the oldest galaxies are associated with the oldest haloes. For models with strong correlations between halo and stellar age, the conformity is too large to be consistent with the data. Weaker implementations of the age-matching model would not produce a detectable signal in SDSS data. We reproduce the results of Kauffmann et al., in which the star formation rates of neighbour galaxies are reduced around primary galaxies when the primaries are low star formers. However, we find this result is mainly driven by contamination in the isolation criterion; when removing the small fraction of satellite galaxies in the sample, the conformity signal largely goes away. Lastly, we show that small conformity signals, i.e. $2-5$ per cent differences in the quenched fractions of neighbour galaxies, can be produced by mechanisms other than halo assembly bias. For example, if passive galaxies occupy more massive haloes than star-forming galaxies of the same stellar mass, a conformity signal that is consistent with recent measurements from PRIMUS (Berti et al.) can be produced.
\end{abstract}

Key words: galaxies: evolution-galaxies: haloes.

\section{INTRODUCTION}

Galaxy evolution is indelibly linked to the evolution of the dark matter structure in which galaxies form. The purpose of this series of papers is to quantify the degree of correlation between galaxy properties and halo properties in the local universe, and through this investigation make inferences about the correlated evolutionary histories of both. The key tool that we use in this series is a galaxy group finder, which, when applied to a statistical sample of galaxies,

\footnotetext{
${ }^{\star}$ E-mail: jeremy.tinker@nyu.edu
}

$\dagger$ Caltech-Carnegie Fellow. can robustly determine which galaxies are central, meaning they exist at the centre of a distinct dark matter halo, and those galaxies that are satellites, meaning they orbit within a larger dark matter halo.

In Paper I of this series (Tinker et al. 2016), we measured the quenched fraction of central galaxies as a function of large-scale environment. The correlation between large-scale density and galaxy properties is well known: galaxies in denser environments are preferentially quenched of their star formation and elliptical in their morphology (see e.g. Blanton \& Moustakas 2009 and references therein). However, when broken down into the relative contribution of central and satellite galaxies, the quenched fraction of central galaxies is nearly independent of environment. The observed 
correlations can be explained by the increasing fraction of satellite galaxies at high densities, which are preferentially quenched (Tinker, Wetzel \& Conroy 2011). We used the conditional abundance matching framework of Hearin \& Watson (2013) and Hearin, Watson \& van den Bosch (2015) to compare these data to models in which halo age is matched to galaxy age at fixed galaxy and halo masses: the older haloes contain the quenched galaxies, while the most rapidly growing haloes contain the most actively star-forming galaxies. This application of the conditional abundance matching framework is known as the 'age-matching' model (Hearin \& Watson 2013). The interest in the age-matching model centres on the fact that the model makes testable predictions for the spatial clustering of active and passive galaxies: at fixed mass, older haloes are more strongly clustered than their younger counterparts, an affect known as assembly bias (see, e.g. Wechsler et al. 2006; Gao \& White 2007; Li, Mo \& Gao 2008). Thus, the galaxies that occupy these haloes - the central galaxies - would show a clear correlation between their quenched fraction and their large-scale density, with most quenched central galaxies being in high-density regions. In the age-matching model, this correlation is expected to be strongest for lower mass galaxies, where assembly bias in dark matter haloes is strongest. In Paper I, we found that the observations were not consistent with predictions of the age-matching model at $M_{*} \lesssim 10^{10.3} \mathrm{M}_{\odot}$. We compared these measurements to a wide variety of halo age definitions. At higher galaxy masses, there was a weak trend of $f_{\mathrm{Q}}^{\text {cen }}$ with $\rho$, a correlation consistent with an age-matching model in which halo age was defined in such a way as to minimize assembly bias within the halo population. The implication of these results is that the mechanism that quenches galaxies is uncorrelated with halo formation history at low masses, and only weakly correlated at higher masses.

In this paper, we probe a complementary observable for detecting assembly bias within the galaxy population: galactic conformity. Galactic conformity is the observed correlation between the properties of separate galaxies. Using group catalogues, Weinmann et al. (2006a) noted that the colours of satellite galaxies within the group were more likely to 'conform' to the colour of the central galaxy at fixed halo mass. These measurements have been confirmed by a number of other studies (Knobel et al. 2015; Kawinwanichakijet al. 2016; Berti et al. 2016). Kauffmann et al. (2013, hereafter K13) measured conformity between the star formation rates of galaxies separated by up to $5 \mathrm{Mpc}-$ well outside the virial radius of the primary galaxy's halo. This large-scale conformity has been proposed as a test of galaxy assembly bias (Hearin et al. 2015). The results of Paper I indicate that galaxy quenching is, at most, weakly correlated with large-scale environment and, by extension, halo formation history. In this paper, we will present new measurements of galactic conformity, as well as a critical examination of the K13 result. Additionally, we will explore sources of a 'conformity signal' that do not arise from assembly bias.

In this work, as in Paper I, we focus on samples of central galaxies in narrow bins of stellar mass. Because the goal is to determine whether halo assembly bias has an impact on galaxy formation, defining the problem in this manner minimizes possible systematic biases in the measurements. We will test for conformity in two different properties of galaxies: galaxy quenched fraction (as in Paper I) and galaxy specific star formation rate (sSFR, as used in Kauffmann et al. 2013). In the latter, we focus on reproducing the K13 measurement and explaining the result in the context of our group catalogues.

\section{DATA, MEASUREMENTS, AND METHODS}

\subsection{Galaxy groups from DR7}

To construct our galaxy samples, we use the NYU Value-Added Galaxy Catalog (VAGC; Blanton et al. 2005) based on the spectroscopic sample in Data Release 7 (DR7) of the Sloan Digital Sky Survey (SDSS; Abazajian et al. 2009). The details of these catalogues, and the algorithm for finding the groups, can be found in Tinker et al. (2011), Campbell et al. (2015), and Paper I. In brief, we create volume-limited samples that are complete in stellar mass, within which the groups are identified. The group finding algorithm assigns probabilities to each galaxy quantifying the likelihood that a galaxy is a satellite, $P_{\text {sat }}$. To create the full central-satellite breakdown of the entire galaxy population, galaxies with $P_{\text {sat }}<0.5$ are classified as central. However, at this threshold for central classification, there are impurities in the sample. To attenuate this effect, we will use galaxies with $P_{\text {sat }}<0.01$. We will refer to these objects as 'pure centrals'. This restriction yields only a modest reduction of the number of centrals in the sample; $\sim 90$ per cent of centrals in the sample are pure. This extra restriction removes central galaxies from the primary sample that are within the projected radius of a larger group but separated in $\Delta v$ by values larger than one to two times the velocity dispersion of the larger halo, depending on how close to the radial edge of the larger halo it lies. This is a primary source of impurities in the central galaxy sample, and restricting our sample to pure centrals increases the purity of the sample to $\sim 99$ per cent.

We use stellar masses from the NYU VAGC, which are in turn created by the code of Blanton \& Roweis (2007). We use $D_{\mathrm{n}} 4000$ as our proxy for identifying galaxies quenched of their star formation. $D_{\mathrm{n}} 4000$, taken from the MPA-JHU SDSS spectral reductions ${ }^{1}$ (Brinchmann et al. 2004), is a more robust indicator of galaxy quiescence because it is less susceptible to dust contamination than broad-band colours (e.g. Maller et al. 2009; Masters et al. 2010). We define a galaxy as quenched if $D_{\mathrm{n}} 4000>1.6$, a value that robustly marks the minimum between the bimodal distribution between the red sequence and the star-forming main sequence.

\subsection{Mock galaxy samples and the age-matching model}

In this paper, we will compare the results from the group catalogue to expectations from dark matter haloes. For most results, we use the 'Chinchilla' simulation, the same simulation used in Paper I. The box size is $400 \mathrm{~h}^{-1} \mathrm{Mpc}$ per side, evolving a density field resolved with $2048^{3}$ particles, yielding a mass resolution of $5.91 \times 10^{8}$ $h^{-1} \mathrm{M}_{\odot}$. The cosmology of the simulation is flat $\Lambda$ cold dark matter, with $\Omega_{m}=0.286, \sigma_{8}=0.82, h=0.7$, and $n_{\mathrm{s}}=0.96$. As in Paper I, haloes are found in the simulation using the ROCKSTAR code of Behroozi, Wechsler \& Wu (2013) and CONSISTENT-TREEs (Behroozi et al. 2013) is used to track halo growth. In Section 3.3, we will use the MultiDark Planck simulation (Klypin et al. 2016) to create mock galaxy samples at $z \sim 0.3$. We will discuss the pertinent details of that simulation and its usage in that section.

In Paper I, we compared measurements directly to statistics of haloes. Here, we create full mock galaxy catalogues that are processed through the group finding algorithm to incorporate and test any observational biases that arise in this procedure. To assign central galaxies to each halo, we do the following: first, we use the

\footnotetext{
${ }^{1}$ http://www.mpa-garching.mpg.de/SDSS/DR7/
} 
results of the observed group catalogue to determine the relationship between host halo mass and central galaxy stellar mass for $M_{*} \geq 10^{9.7} \mathrm{M}_{\odot}$. Haloes in the simulation are matched to haloes of the same mass in the group catalogue, thus any scatter found in the group catalogue is preserved in the mock. Once the stellar masses of the central galaxies have been assigned, the mock central galaxies and the group catalogue central galaxies are divided into bins of 0.1 dex of stellar mass. In each bin, the mock central galaxies are rank ordered by the age of their haloes (which we will define below). Once ranked, values of $D_{\mathrm{n}} 4000$ are assigned to the mock central galaxies by matching the rank-ordered lists of halo age to group catalogue $D_{\mathrm{n}} 4000$ : the oldest halo is assigned the highest value of $D_{\mathrm{n}} 4000$, the second oldest halo gets the second highest $D_{\mathrm{n}} 4000$, and on down the lists. This method is consistent with the age-matching model of Hearin \& Watson (2013) and yields a conformity signal similar to those presented in Hearin et al. (2015). We also have a mock with no assembly bias, in which $D_{\mathrm{n}} 4000$ values are assigned randomly in each bin of stellar mass. In this latter model, there should be no conformity signal because the probability of being quenching is uncorrelated with the halo age.

To assign satellites to each halo, we first measure the mean number of satellites at $M_{*} \geq 10^{9.7} \mathrm{M}_{\odot}$ as a function of halo mass in the SDSS group catalogue. For each halo in the simulation, we randomly draw a Poisson deviate around the mean to represent the number of satellites in that halo, $N_{\text {sat }}$. From the group catalogue, we bin all satellites by their host halo mass. For each simulated halo, we randomly draw actual satellites from the halo mass bin corresponding to that halo, up to $N_{\text {sat }}$. Thus, each satellite in the simulated haloes has the values of $M_{*}$ and $D_{\mathrm{n}} 4000$ from the SDSS satellite. There is no assembly bias in the satellite galaxies - i.e. whether a satellite is star forming or quenched is independent of the properties of the host halo. As shown in Hearin et al. (2015), satellite assembly bias has minimal - if any - impact on large-scale galactic conformity.

We define halo age using various definitions, all of which are discussed in detail in Paper I. These age definitions fall into two distinct classes: (1) halo growth over a redshift baseline, and (2) proxies for 'formation epoch' of the haloes. For (1), our fiducial model rank orders haloes by their growth since $z=0.8$. We also investigate other baselines using $z=0.2,0.4$, and 1.5. A redshift baseline of $z=0.8 \rightarrow 0$ roughly spans the range over which most $M_{*} \lesssim 10^{11.3} \mathrm{M}_{\odot}$ central galaxies arrive on the red sequence (Tinker et al. 2013). Time-scales from lower redshifts reflect short-term growth, near time-scales estimated for the quenching time-scale of galaxies (Peng, Maiolino \& Cochrane 2015; Hahn, Tinker \& Wetzel 2016). Longer baselines are closer to the half-mass redshifts of $M_{\mathrm{h}} \sim 10^{12} \mathrm{M}_{\odot}$ haloes. For (2), we use the half-mass redshift itself, $z_{1 / 2}$, which is the most common age definition in the literature. We also use two different versions of the formation epoch defined in Wechsler et al. (2002), $a_{\mathrm{c}}$, which we describe presently.

The typical implementation of $z_{1 / 2}$ or $a_{\mathrm{c}}$ uses the redshift evolution of the halo itself, $M_{\mathrm{h}}(z)$. However, this quantity is not always monotonically increasing. Tidal encounters with larger haloes, or even 'splashback' events, in which haloes actually pass through a larger halo and emerge back out, can strip mass off the halo. Thus, $M_{\mathrm{h}}$ at $z=0$ may be lower than the peak halo mass, $M_{\text {peak }} \cdot M_{\text {peak }}(z)$ is defined as the highest halo mass at any time $\geq z$, and it is a monotonically increasing function of time. Haloes that have encountered significant stripping will be ranked very high when identifying the 'oldest' objects. In Paper I, we showed that this is what drives the very strong assembly bias signal in low-mass haloes. Using halo growth histories as a function of $M_{\text {peak }}(z)$ rather than $M_{\mathrm{h}}(z)$ removes the effects of these types of encounters. In Wetzel et al. (2014), we demonstrated that splashback encounters have little immediate impact on the galaxy star formation rate; such objects quench the same as satellite galaxies, in that there is a delay of several Gyr before any quenching of star formation begins. Thus, in Paper I, we concluded that a more physically realistic age-matching model should use $M_{\text {peak }}(z)$ rather than $M_{\mathrm{h}}(z)$ to determine halo age. The $M_{\text {peak }}$ age-matching model was in good agreement with measurements of the dependence of $f_{\mathrm{Q}}$ on large-scale density for high-mass galaxies $\left(M_{*} \gtrsim 10^{10.3} \mathrm{M}_{\odot}\right.$, where the $f_{\mathrm{Q}}$ for central galaxies goes above 50 per cent). However, even $a_{\mathrm{c}}\left(M_{\text {peak }}\right)$ produced a correlation between $f_{\mathrm{Q}}$ and $\rho$ much stronger than that measured in galaxies at lower stellar masses.

After populating the haloes with mock galaxies, the galaxies are projected into an angular space, giving each galaxy an RA, Dec., and $z$, covering a total area of $5156 \mathrm{deg}^{2}$ (1/8 of the sky) and extending to a maximum redshift of $z=0.138$, which corresponds to a comoving radius of the box length, $400 \mathrm{~h}^{-1} \mathrm{Mpc}$. Each mock is then passed through the group finder. All conformity measurements from the mocks are measured on the mock group catalogues in order to incorporate any biases imparted by the group-finding process (see Campbell et al. 2015, for a thorough assessment of the precision and accuracy of the group finder used here as well as other finders in the literature). Additionally, the group finding process does not use $D_{\mathrm{n}} 4000$ information at all, only positions, velocities, and stellar masses. Thus, even if the assignment of $D_{\mathrm{n}} 4000$ values is biased, this does not impact the resulting group catalogue.

\subsection{Making conformity measurements}

To quantify conformity, we measure the fraction of 'secondary' galaxies that are quenched, $f_{\mathrm{Q}}$, as a function of projected separation, $R_{\mathrm{p}}$, from 'primary' galaxies. Primary galaxies are divided into quenched or star-forming samples. We will refer to $f_{\mathrm{Q}}$ around each type of primary galaxy as $f_{\mathrm{Q}}^{\text {red }}$ and $f_{\mathrm{Q}}^{\text {blue }}$, respectively. To be clear, primary and secondary samples do not imply mutually exclusive sets of galaxies. In our fiducial measurements, secondary galaxies are defined as central galaxies of the same mass range as the primary galaxies, but we will show examples of results when including satellite galaxies in the secondary sample. Primary galaxies can be a secondary to another primary. We will show presently that our choices of primary and secondary galaxies remove observational biases.

At each bin in $R_{\mathrm{p}}$, we include galaxies with $\Delta v \leq 500 \mathrm{~km} \mathrm{~s}^{-1}$ with respect to the primary galaxy. We make all measurements in bins of fixed stellar mass. Our goal in this paper is to use conformity as a test of assembly bias. Halo assembly bias is the effect that the clustering of galaxies at fixed halo mass depends on halo formation history. Restricting the galaxy sample to only centrals brings the sample closer to a sample of host haloes. Performing the measurements in bins of fixed stellar mass is a rough approximation for fixing halo mass.

Fig. 1 shows and example of our conformity measurements for $\log M_{*} / \mathrm{M}_{\odot}=[9.7,9.9]$ for both mock galaxies and the SDSS group catalogue (we will discuss the SDSS measurements in detail in Section 3). The top two panels show the results measured from the SDSS group catalogues, while the bottom two panels show results from our mock galaxy catalogues. The left-hand panels show results when the primary sample is made up of all central galaxies, and the right-hand panels show results when the primary sample is restricted to pure centrals. In each panel, we show $f_{\mathrm{Q}}$ where the secondaries are centrals (bottom), satellites (top), and all galaxies (middle). 

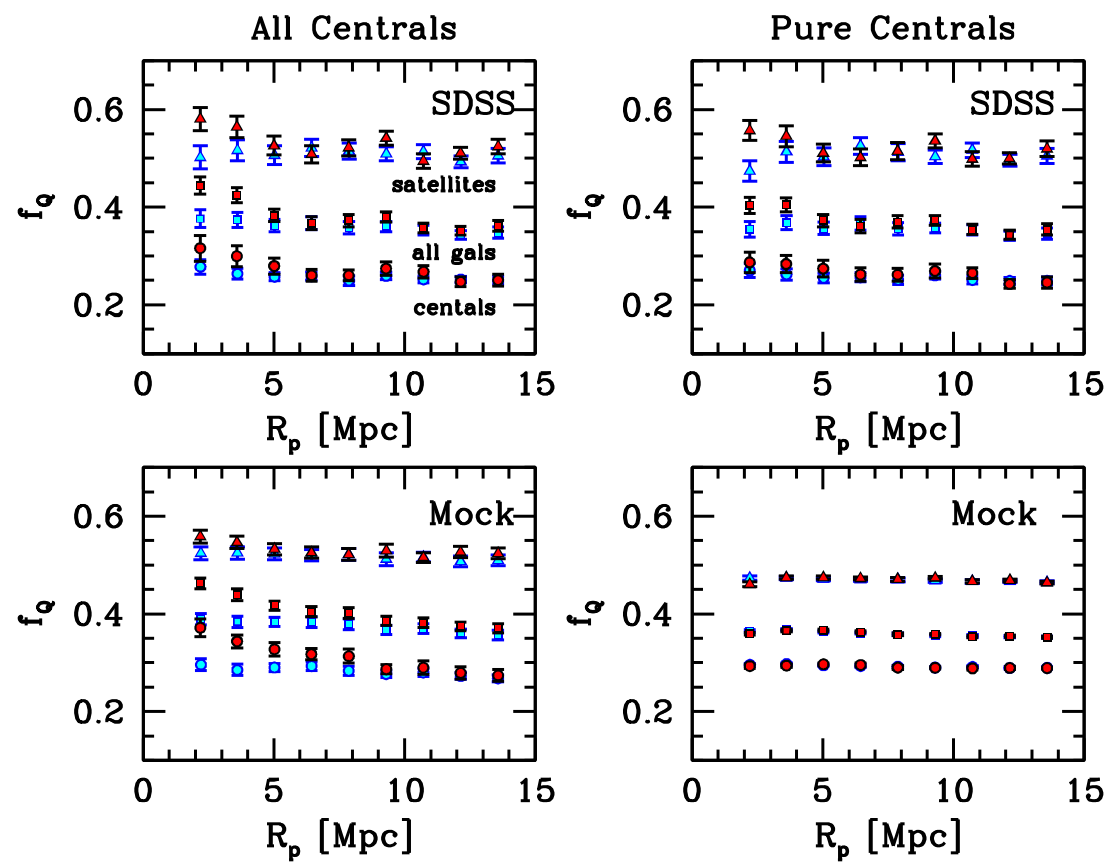

Figure 1. The conformity signal around both mock galaxy catalogues and SDSS galaxies of mass $M_{*}=10^{10} \mathrm{M}_{\odot}$, in a bin 0.2 dex wide. We show $f_{\mathrm{Q}}$ of secondary galaxies around primary central galaxies as a function of projected separation. In each panel, from top to bottom, the secondary galaxies are satellite galaxies (triangles), all galaxies (squares), and central galaxies (circles). Assembly bias, if present, should primarily affect $f_{\mathrm{Q}}$ for central secondaries. Blue symbols represent $f_{\mathrm{Q}}$ around primaries that are star forming, while red symbols represent primaries that are quenched. The left-hand panels show the results when the sample of primary galaxies includes all central galaxies of $10^{10} \mathrm{M}_{\odot}$, while the right-hand panels show the results when the primary sample is restricted to 'pure' central galaxies, which are centrals with a $P_{\text {sat }}<0.01$. The mock galaxy catalogue has no assembly bias in it, thus the conformity 'signal' seen on the left-hand side is purely an artefact of impurities in the group finder. This bias is gone when restricting the primaries to pure centrals.

We first discuss the mock results. The mock catalogue used in Fig. 1 contains no assembly bias; i.e. $D_{\mathrm{n}} 4000$ is uncorrelated with any halo age proxy. Thus, the mock contains no intrinsic galactic conformity, and any difference in $f_{\mathrm{Q}}^{\text {red }}$ and $f_{\mathrm{Q}}^{\text {blue }}$ is due entirely to biases from the group finder. Thus, the rise in $f_{\mathrm{Q}}^{\text {red }}$ for all centrals is due to misclassification of centrals and satellites within the primary galaxy sample. This is an example of observational biases that can result from how primary galaxies are identified. However, when restricting the primaries to pure centrals, all biases are eliminated. The results for $f_{\mathrm{Q}}^{\text {red }}$ and $f_{\mathrm{Q}}^{\text {blue }}$ are independent of $R_{\mathrm{p}}$ and consistent with each other.

The results for the SDSS group catalogue are quantitatively similar to the mock results. When using all centrals as the primary sample, the quenched fraction around red primaries rises as $R_{\mathrm{p}}$ approached the virial radius of the halo, diverging from the quenched fraction around star-forming primaries. These trends are largely removed when shifting the primary sample to pure centrals. We will discuss these results more quantitatively in Section 4.

\subsection{The detectability of assembly bias with galactic conformity}

Fig. 2 shows measurements of conformity for all our various definitions of halo age. From this point forward, all results use primary galaxies that are pure central galaxies and secondary galaxies are all central galaxies in the same stellar mass bin. In both panels, we show our fiducial model using halo growth since $z=0.8$ with the solid curve with error bars. Error bars are obtained by jackknife sampling based on RA and Dec. The left-hand side of Fig. 2 shows the results for age-matching modelling in which haloes are ranked by fractional growth over various timespans. For any definition, there is a clear conformity signal. In fact, at small scales, $R_{\mathrm{p}} \lesssim 3$
$\mathrm{Mpc}$, the conformity signal is independent of redshift baseline. This is likely because haloes that have recently had a tidal interaction will appear as low-growth haloes in all models, but for short time-scales, the haloes that interacted with each other will still be in proximity with one another. At larger scales, there is a clear monotonic trend of a larger conformity signal with longer redshift baseline, saturating for $z \gtrsim 0.8$. For $z=0.2 \rightarrow 0$, there is almost no conformity past $R_{\mathrm{p}}=5 \mathrm{Mpc}$.

In the right-hand panel of Fig. 2, we show the various formation epoch definitions. For $z_{1 / 2}$ and $a_{\mathrm{c}}\left(M_{\mathrm{h}}\right)$, the conformity signal is roughly consistent and somewhat smaller than the maximal effect seen for $z=0.8$ halo growth. However, when using $a_{\mathrm{c}}\left(M_{\text {peak }}\right)$, the amplitude of the conformity signal is attenuated at all scales, including small scales where recent tidal interactions come into play. Because tidal interactions do not alter a halo's $M_{\text {peak }}$ value, the small-scale two-halo conformity signal is much smaller.

\section{RESULTS}

\subsection{Galactic conformity in $f_{\mathrm{Q}}$ for central galaxies}

Fig. 3 presents our measurements of $f_{\mathrm{Q}}$ around pure central galaxies in the SDSS group catalogues, shown with the blue and red symbols. Each panel shows results from a different stellar mass bin. All are 0.2 dex wide. Recall that both primaries and secondaries are restricted to the same stellar mass, and secondary galaxies are centrals only. Error bars on the SDSS measurements are obtained by dividing the sky area into 25 roughly equal patches of sky and performing jackknife sampling. The size of each patch is determined such that the number of galaxies in each patch is the same. The choice of 25 balances the need for more patches than data points, while still 

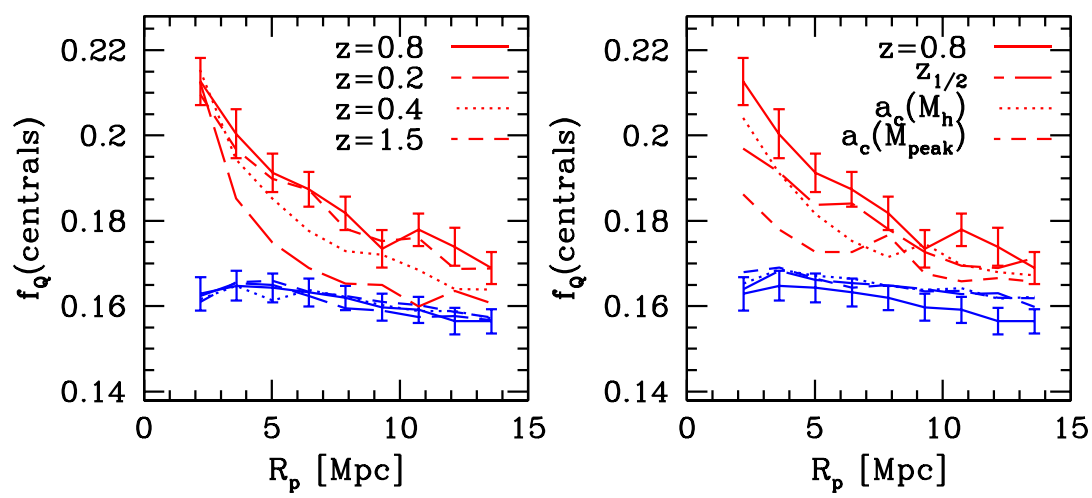

Figure 2. The conformity signal in a bin of $\log M_{*} / \mathrm{M}_{\odot}=$ [9.7-9.9] for different implementations of the age-matching model. All results are obtained after running the group finder on each mock and analysing the resulting mock group catalogue. All results use a primary sample of pure centrals and a secondary sample of all centrals. Left-hand panel: results of age-matching models where halo age is defined as fractional growth over a redshift baseline. Haloes with the least amount of growth over this time frame are ranked as the oldest. Right-hand panel: results of age-matching models where age is defined as the half-mass redshift, $z_{1 / 2}$, (long dash), the formation epoch of Wechsler et al. (2002), $a_{\mathrm{c}}\left(M_{\mathrm{h}}\right)$ (dotted), and the formation epoch using $M_{\text {peak }}(z)$ (short dash) rather than the current mass, $M_{\mathrm{h}} . M_{\text {peak }}$ is always monotonically increasing by construction.
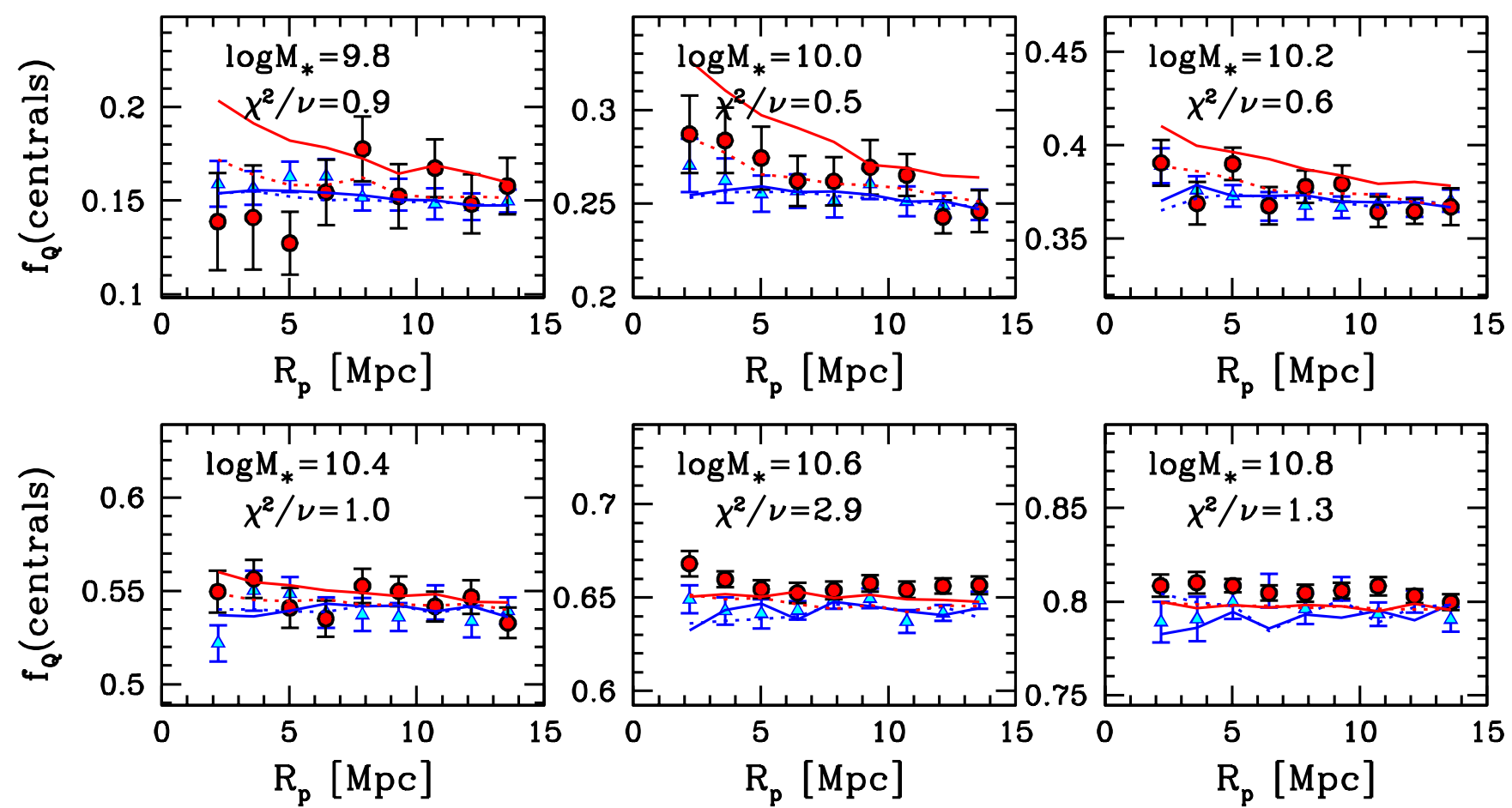

Figure 3. Conformity signal around primary galaxies as a function of stellar mass. In each panel, the primaries are pure centrals, while secondaries are all centrals of the same stellar mass. The symbols represent measurements from the SDSS group catalogue, while solid curves show predictions from the age-matching model using $\Delta z=0.8$ as the age proxy. Dotted curves show the age-matching predictions for $a_{\mathrm{c}}\left(M_{\text {peak }}\right)$. Red symbols and curves show $f_{\mathrm{Q}}$ around quenched primaries, while blue symbols and curves show $f_{\mathrm{Q}}$ around star-forming primaries. Error bars are obtained by jackknife sampling. In each panel, the value of $\chi^{2} / v$ is obtained by comparing $f_{\mathrm{Q}}^{\text {blue }}$ and $f_{\mathrm{Q}}^{\text {red }}$. Values over unity indicate a statistically significant difference between the two quantities, and thus imply a detection of conformity.

having patches that are large enough that each region is (mostly) uncorrelated. The value of $\chi^{2} / v$ in each panel is obtained comparing $f_{\mathrm{Q}}^{\text {red }}$ and $f_{\mathrm{Q}}^{\text {blue }}$,

$\chi^{2} / v=\frac{1}{N_{\text {data }}} \sum \frac{\left(f_{\mathrm{Q}}^{\text {red }}-f_{\mathrm{Q}}^{\text {blue }}\right)^{2}}{\sigma_{\text {red }}^{2}+\sigma_{\text {blue }}^{2}}$,

where $v=N_{\text {data }}$ is the number of data points (9), and $\sigma_{\text {red }}$ and $\sigma_{\text {blue }}$ represent the errors on $f_{\mathrm{Q}}^{\text {red }}$ and $f_{\mathrm{Q}}^{\text {blue }}$, respectively. For five of the six stellar mass bins, there is no statistically significant evidence for a difference between $f_{\mathrm{Q}}^{\text {red }}$ and $f_{\mathrm{Q}}^{\text {blue }}$. The lone exception is the conformity measurement for galaxies with $\log M_{*} / \mathrm{M}_{\odot}=[10.5$, 10.7], which we will discuss subsequently.

In each panel, we show the predictions for two different agematching models: the $z=0.8$ and the $a_{\mathrm{c}}\left(M_{\text {peak }}\right)$ models. At low stellar masses, the differences between these two models is especially clear, with $z=0.8$ producing a clearer signal, larger than that measured in the data. At masses above $M_{*}=10^{10.3} \mathrm{M}_{\odot}$, the predictions of both models show only modest, if any, conformity. 

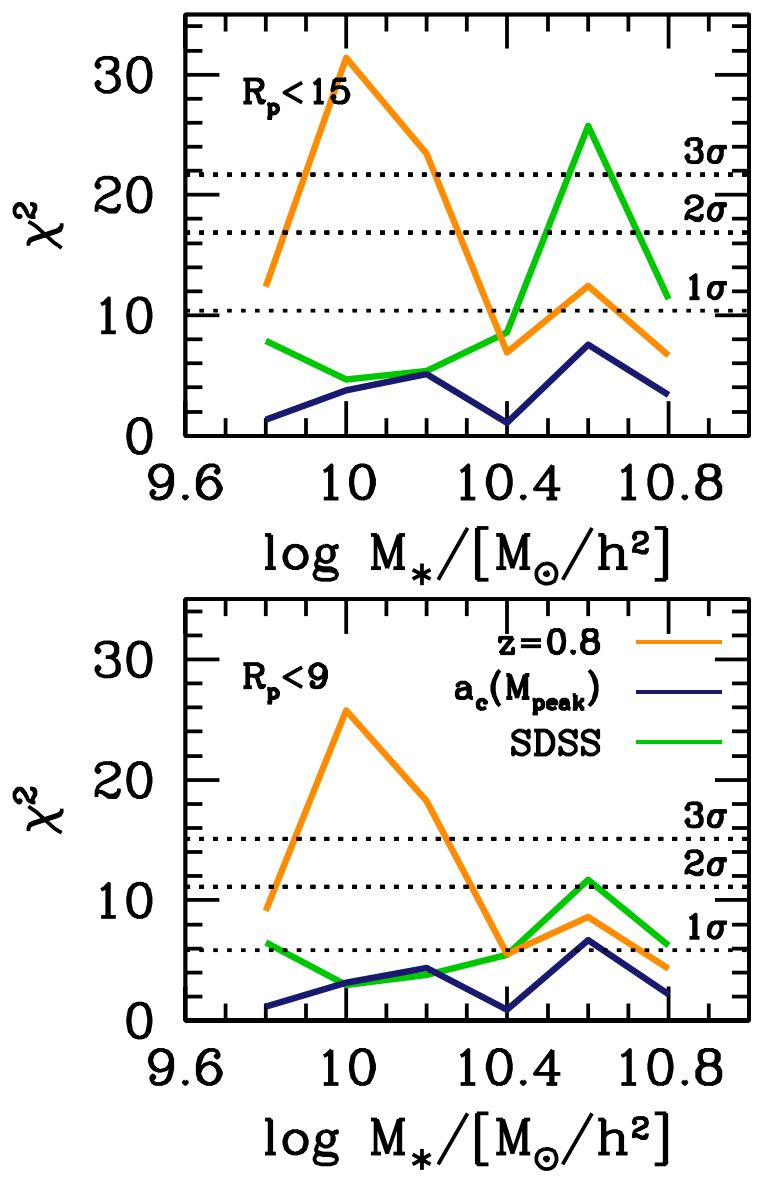

Figure 4. Top panel: $\chi^{2}$ values, as defined by equation (1), showing the difference in $f_{\mathrm{Q}}^{\text {red }}$ and $f_{\mathrm{Q}}^{\text {blue }}$ as a function of $M_{*}$. The three solid curves show results for the SDSS data, the $z=0.8$ age-matching model, and the $a_{\mathrm{c}}\left(M_{\text {peak }}\right)$ model. The dotted curves show confidence levels from a $\chi^{2}$ distribution for 9 degrees of freedom, representing the number of $R_{\mathrm{p}}$ bins in the measurement. For the age-matching models, the errors are taken from the data, not from the mocks, which are significantly larger. Thus, the curves represent the $\chi^{2}$ that would be obtained if the model's conformity signal were present in the data. Bottom panel: same as the top panel, but now restricting all $\chi^{2}$ values to $R_{\mathrm{p}}<6 h^{-1} \mathrm{Mpc}$ bins ( 5 degrees of freedom). Note that the $>3 \sigma$ result from $M_{*}=10^{10.3} \mathrm{M}_{\odot}$ is attenuates when removing larger scales, implying that the result is partially driven by a statistical fluctuation or that it is dependent on scale in a manner not seen in any theoretical model.

Fig. 3 raises two pertinent questions: (1) what are the $\chi^{2}$ values if we restrict our measurement to smaller scales, where the conformity signal is predicted to be clearest, and (2) if the conformity signal in the data were as strong as the age-matching models, would we have been able to detect it given the larger errors in the data?

Both of these questions are addressed in Fig. 4. In the top panel, we show $\chi^{2}$ from equation (1) - without dividing by the degrees of freedom - for the SDSS data as a function of stellar mass. For reference, the $1 \sigma, 2 \sigma$, and $3 \sigma$ confidence levels from a $\chi^{2}$ distribution for 9 degrees of freedom are shown with the horizontal dashed lines. The other two lines represent the $\chi^{2}$ values obtained from the $z=0.8$ and $a_{\mathrm{c}}\left(M_{\text {peak }}\right)$ models after replacing the error bars on those predictions with the error bars obtained from the SDSS group catalogues. Thus, a $\chi^{2}$ above 16.5 indicates that, if the amount of assembly bias seen in the age-matching model were present in the SDSS data, it would be detectable at 95 percent confidence. Unsurprisingly, for the $a_{\mathrm{c}}\left(M_{\text {peak }}\right)$ age-matching model, the amount of conformity induced is too weak to be detected at any stellar mass. The $z=0.8$ model yields a $>3 \sigma$ signal for lower stellar masses, where the assembly bias in dark matter haloes is strongest. The $\chi^{2}$ value for the smallest stellar mass bin is barely above $1 \sigma$, owing to the small volume of this sample.

The lower panel of Fig. 4 shows the $\chi^{2}$ for the same models and data, only now we exclude the data points at $R_{\mathrm{p}}=9 \mathrm{Mpc}$ and above, reducing the number of degrees of freedom to 5 . Relative to the confidence levels, the results are consistent with those of the full measurement. However, we note that the lone $>3 \sigma$ detection in the data, at $M_{*}=10^{10.6} \mathrm{M}_{\odot}$, is now reduced to $\approx 2 \sigma$. In all models of conformity, the signal is larger at smaller separations. For the statistical significance to reduce when excluding larger scales argues that this is a fluctuation, or due to some effect that is distinct from galaxy assembly bias.

\subsection{Conformity in the star formation rates of galaxies}

How can our results be reconciled with the measurements of K13? They are substantially different measurements, given our use of group catalogues to identify centrals, and our restriction of the secondary population to be centrals of the same stellar mass as the primaries, and the 'quantity of conformity' being quenched fraction rather than median sSFR. First, we reproduce the K13 measurement, then explain the differences in our results. We will demonstrate that our use of a group catalogue to robustly identify primary galaxies is the key difference in the comparison.

Fig. 5 shows our reproduction of the K13 conformity measurement. For this measurement, all galaxy stellar mass and star formation rates come from the MPA-JHU catalogue as done in K13. In the previous sections of this paper, we use NYU-VAGC stellar masses. K13 also uses a mixture of the total SSFR - corrected for the finite aperture of the fibre relative to the angular size of the galaxy - and the sSFR only within the fibre aperture. We will refer to the total $\mathrm{sSFR}$ as such and the rate within the fibre as $\mathrm{SSFR}^{\text {(fib) }}$. We created a stellar mass complete sample incorporating all galaxies with $0.017<z<0.030$ and $M_{*}>10^{9.25} \mathrm{M}_{\odot}$. Primary galaxies are identified within this sample using the isolation criterion of K13: a galaxy with stellar mass $M$ is isolated if there are no galaxies more massive than $M / 2$ within a projected separation of $500 \mathrm{kpc}$ and a $\Delta v$ of $500 \mathrm{~km} \mathrm{~s}^{-1}$. Secondary galaxies are all galaxies within the sample. The primary galaxies are broken in percentile bins based upon their total sSFR: 0-25 per cent, $25-50$ per cent, $50-75$ per cent, $>75$ percent, and $>90$ percent. The $y$-axis represents the median $\mathrm{SSFR}^{(\mathrm{fib})}$ for secondary galaxies around each bin in primary sSFR. The left-hand panel in Fig. 5 compares our measurement to that of K13: all primaries and secondaries are used. Error bars are from spatial jackknife subsampling of the SDSS footprint into 25 equal-area regions. There are slight differences in the median $\mathrm{SSFR}^{(\mathrm{fib})}$ for the lowest star-forming primaries, but the results are consistent in general and, in particular, both measurements show a strong conformity signal; neighbouring galaxies around primaries with suppressed star formation rates also show significantly lower sSFRs. We find similar comparison to K13 when using aperture-corrected sSFR.

We have also run the group finder on this catalogue, enabling us to investigate the agreement between K13's isolation criterion and our own, and to bring these measurements into a more common framework with the conformity measurements of the quenched fraction earlier in the paper. In the group catalogue, 3.5 per cent of the K13 primary galaxies are classified as satellites. Fig. 6 shows several examples of galaxies that are classified as isolated according to K13 but are denoted as satellite galaxies in the group finder. 


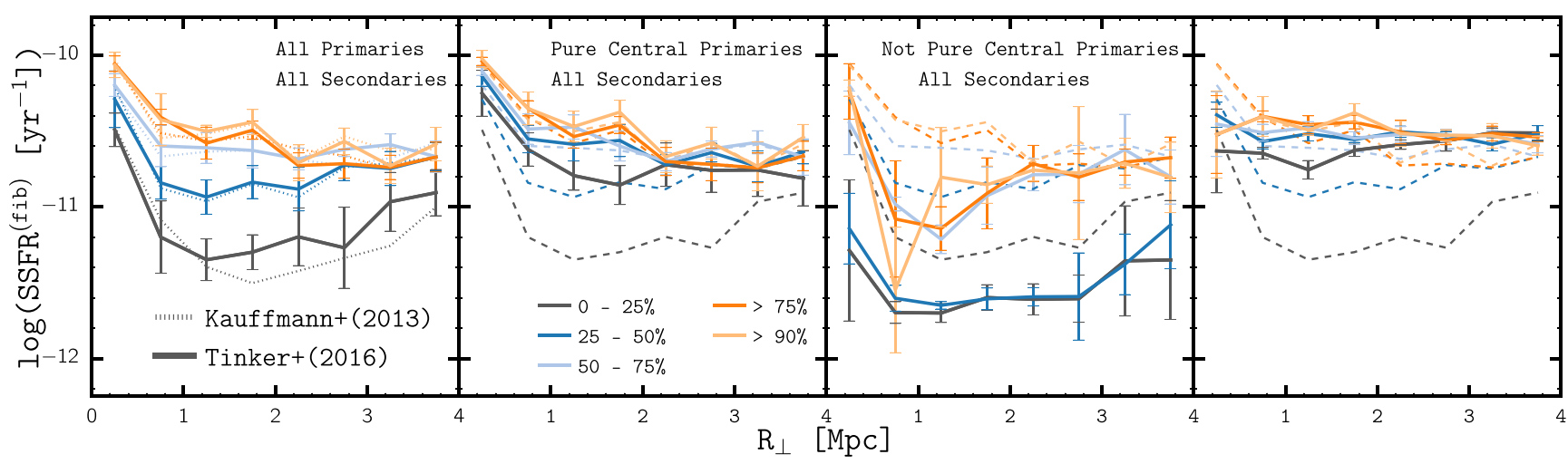

Figure 5. Our reconstruction and subsequent deconstruction of the K13 conformity measurement. Panels from left to right: leftmost panel: the original K13 measurement: the median SSFR within the SDSS fibre, sSFR ${ }^{(\mathrm{fib})}$, of secondary galaxies around primary galaxies isolated with the K13 criterion. Primary galaxies are in the stellar mass range $\log M_{*} / \mathrm{M}_{\odot}=[10.0,10.5]$, while secondary galaxies include all galaxies in the sample. The thin solid curves are taken from K13, and the thick curves with error bars are our own measurement. The error bars are obtained by spatial jackknife of the sample. Second panel: thick solid lines show the measurement after removing from the primary sample galaxies that are classified by the group finder as either satellite galaxies are non-pure satellites. This removes $\sim 6$ per cent of galaxies from the primary sample. The dotted curves - here and in the other right-hand panels - show our measurement of conformity from the leftmost panel. Third panel: the conformity signal of the galaxies that were removed from the primary sample in the second panel. Half of these galaxies are classified as satellites, while the other half are classified as low-probability centrals. Right-hand panel: the conformity signal when restricting the secondary galaxies to be central galaxies of the same stellar mass as the primary sample. The volume of this catalogue is larger than the other three panels, thus the error bars are smaller.
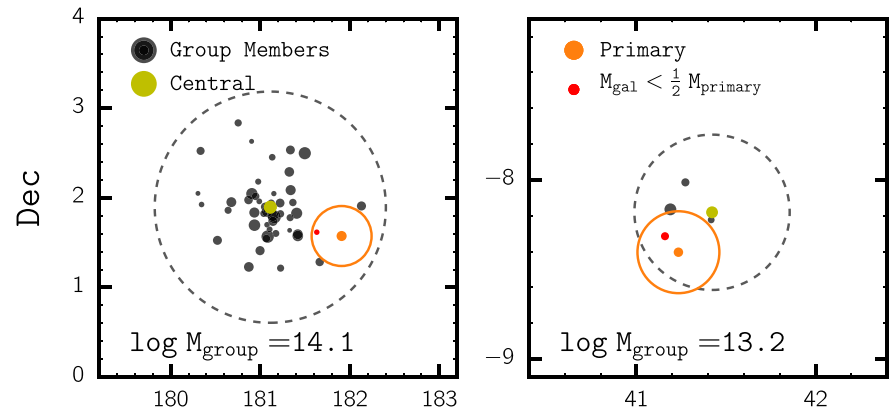
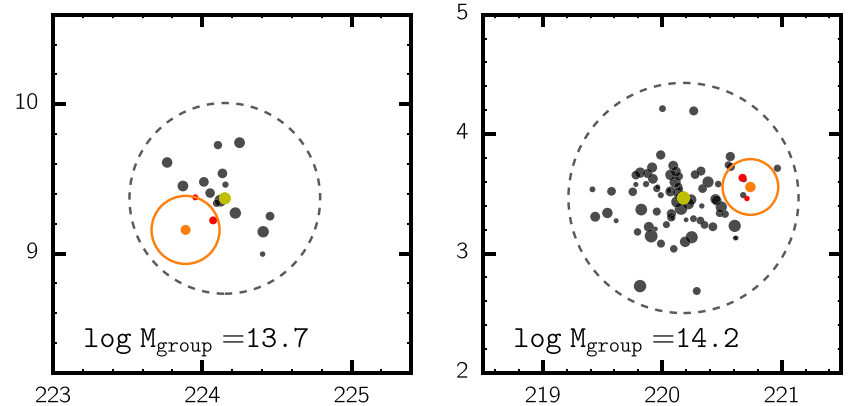

RA

Figure 6. Examples of galaxies classified as isolated by the K13 criteria, but are marked as satellites within a group by the group finder. In each panel, the isolated primary galaxy in question is marked in orange, with the isolation radius shown with the orange circle. The group virial radius is indicated with the dashed circle, while other group members are shown in grey, with point size proportional to $\log M_{*}$. The group central galaxy is shown in green. Red dots indicate galaxies that are within the projected radius of the isolation criteria but are less massive than $M_{*} / 2$. Other group members (grey symbols) within the isolation radius are outside the velocity separation criterion $\left(\Delta v>500 \mathrm{~km} \mathrm{~s}^{-1}\right)$.

Additionally, another 3 per cent of the K13 primary galaxies are classified as 'non-pure' central galaxies (i.e. their $P_{\text {sat }}$ values are $<0.5$ but $>0.01$ ). These two subpopulations represent only 6.5 per cent of the K13 primary sample, but they have a dramatic impact on the measured conformity signal. The second panel in Fig. 5 shows the conformity measurements for the 93.5 per cent of K13 primaries that are also listed as pure centrals. The conformity signal is nearly gone. The sSFR of secondary galaxies is roughly independent of the SSFR of the primary galaxy, with some small differences at $1<R_{\mathrm{p}}<2 \mathrm{Mpc}$. Because the primary sample is made up of relatively massive galaxies at $\log M_{*} / \mathrm{M}_{\odot}=[10.0,10.5]$, any satellites within this sample will lie in a massive halo. These massive haloes will, in turn, contain a large number of quenched satellite galaxies up to $\sim 2 \mathrm{Mpc}$ away from the satellite-primary galaxy (1 Mpc being the radius of $M_{\mathrm{h}} \sim 10^{14} \mathrm{M}_{\odot}$ haloes). Although the overall fraction of quenched galaxies is $\sim 50$ per cent at $\log M_{*} / \mathrm{M}_{\odot}=[10.0,10.5]$, for satellites at that mass scale, the quenched fraction is $\gtrsim 80$ per cent, thus the inclusion of these galaxies specifically biases the lowest two bins in primary sSFR, as can be seen in Fig. 5.
On the right-hand panel, we attempt an apples-to-apples comparison of the conformity signals made on $f_{\mathrm{Q}}$ to sSFR. The primary galaxies are, once again, pure central galaxies in the stellar mass range $\log M_{*} / \mathrm{M}_{\odot}=[10.0,10.5]$, but now we restrict the secondary galaxies to also be central galaxies within the same mass range. To enhance the statistics in the measurement, we create a new stellar-mass-limited catalogue for galaxies with $M_{*}>10^{10.0} \mathrm{M}_{\odot}$ and $0.017<z<0.0525$, and run the group finder on this catalogue. When constructed in the same manner as the $f_{\mathrm{Q}}^{\text {cen }}$ measurements, the sSFR conformity measurements are consistent.

The group finder is not infallible; from mock tests, roughly 20 per cent of the central galaxies are actually misclassified satellites. However, that number falls to around 1.8 per cent overall, and 1.6 per cent in the mass range for the K13 measurements, when restricting the sample to pure centrals. Whether or not the labelling of a galaxy as 'central' or 'satellites' in the group catalogue is 100 per cent accurate, a conservative interpretation of Fig. 5 is that a more restrictive isolation criterion essentially eliminates the conformity signal seen in K13. Furthermore, any mislabelling of centrals 
and satellites in our primary sample not eliminated by the purity cuts is likely to contribute to a conformity signal, so the results in the right-hand panel of Fig. 5 are upper limits on the true conformity.

\subsection{Conformity from alternate sources}

The results of the previous section indicate that the conformity signal can be sensitive to the details of the isolation criterion. Fig. 7 shows the conformity signal in several different mock galaxy catalogues, none of which contain any galaxy assembly bias (i.e. the values of $D_{\mathrm{n}} 4000$ do not correlate with halo formation history). Here, conformity is measured as the quenched fraction of secondary galaxies around star-forming and quenched primary galaxies. Primaries are identified using the isolation criterion of K13 on galaxies in the stellar mass range $\log M_{*} / \mathrm{M}_{\odot}=[10.6,11.1]$. We choose this mass range because it yields a median galaxy mass close to that recent conformity results from Berti et al. (2016) using PRIMUS data, which also use the K13 isolation criteria. Secondary galaxies are all galaxies in the mock, which is complete down to $M_{*}=10^{9.7}$ $\mathrm{M}_{\odot}$. We note that the results shown in this figure are qualitatively the same when using a sample of primaries in the range $\log M_{*} / \mathrm{M}_{\odot}=[10.0,10.5]$, as in $\mathrm{K} 13$.

The top panel in Fig. 7 shows the results for a mock with no assembly bias. This is the same mock used in Fig. 1 to test our method of measuring conformity using the group catalogue. At $R_{\mathrm{p}}>2 \mathrm{Mpc}$, there is no conformity. However, at smaller scales, there is a small but measurable difference in the quenched fractions of secondary galaxies around star-forming and quiescent primaries. At $R_{\mathrm{p}} \sim 1 \mathrm{Mpc}$, this difference is around 2 per cent, driven mostly by the same effects seen in the K13, in which a small fraction of satellite galaxies make it into the primary sample.

In the middle panel, we incorporate the effects of backsplash galaxies into the mock. As discussed above, backsplash galaxies are those that are currently classified as central, but have in their past history passed through the virial radius of a larger halo. Wetzel et al. (2014) showed that the slight enhancement of the $f_{\mathrm{Q}}$ around groups and clusters can be explained by a model in which backsplash galaxies evolve the same as satellite galaxies: several Gyr after the initial accretion event, the galaxies undergo rapid quenching and migrate on to the red sequence. Most backsplash galaxies are eventually re-accreted back into the larger halo, but some exist as centrals long enough to be quenched, while outside the group or cluster's virial radius. Here, we identify all central galaxies that are backsplash galaxies. If the initial accretion event took place more than $4 \mathrm{Gyr}$ ago, the galaxy is marked as quenched if it is not already. This delay time is taken from the results of Wetzel et al. (2014) to match the observed quenched fraction around groups and clusters. In the mock, to offset the overall increase in $f_{\mathrm{Q}}^{\text {cen }}$, a random sample of quenched central galaxies are reclassified as star forming in order to preserve the initial quenched fraction. This process reclassifies about 5 per cent of the central galaxy population.

Backsplash galaxies are preferentially near large central galaxies. The impact of this on the conformity signal is seen in the middle panel of Fig. 7. There is a slight difference in the quenched fractions all the way out to $5 \mathrm{Mpc}$, caused by splashback galaxies themselves being classified as primaries. But inside $2 \mathrm{Mpc}$, the difference in $f_{\mathrm{Q}}$ around star-forming and quenched primaries monotonically rises to around 4 per cent at $R_{\mathrm{p}} \sim 1 \mathrm{Mpc}$,

The bottom panel shows the results of measuring conformity on a mock constructed from the stellar-to-halo mass relations (SHMR) calibrated on COSMOS data in Tinker et al. (2013). The key difference between this mock and the ones used elsewhere in this paper is

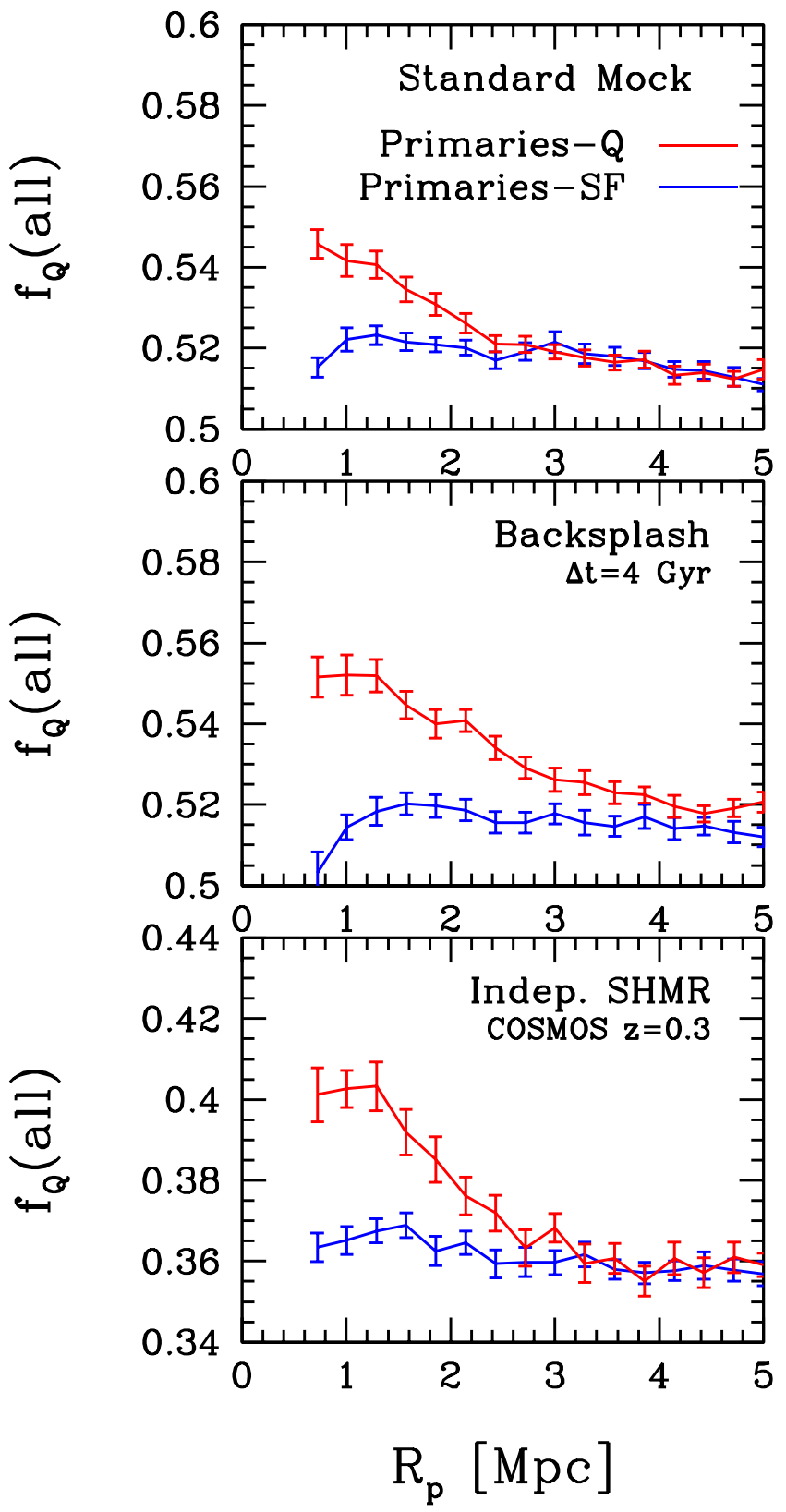

Figure 7. Three measurements of conformity using the K13 isolation criterion on mock galaxy samples. In each panel, the primaries at isolated galaxies in the stellar mass range $\log M_{*} / \mathrm{M}_{\odot}=[10.6,11.1]$, while the secondaries are all galaxies with stellar masses $\log M_{*} / \mathrm{M}_{\odot}>9$.7. Curves show the quenched fraction of secondaries around star-forming and quenched primaries. Error bars are from jackknife sampling. Top panel: mock with no assembly bias. This is the same mock used in Fig. 1. Middle panel: mock using the same galaxy catalogue, but splashback galaxies with first accretion times more than $4 \mathrm{Gyr}$ ago are classified as quenched. See the text for more details. Bottom panel: mock where the stellar-to-halo mass relation of starforming and quenched galaxies are taken from Tinker et al. (2013), in which the halo masses of quiescent galaxies are higher than those of star-forming galaxies at fixed stellar mass. The relations are calibrated to match statistics of galaxies in COSMOS data at $z=0.3$. See the text for further details. In each panel, there is a marginal detection of conformity at $R_{\mathrm{p}}<2 \mathrm{Mpc}$, even though each mock has no assembly bias. 
that Tinker et al. (2013) use stellar mass functions, clustering, and galaxy-galaxy lensing measured separately for passive and starforming galaxies to constrain the relationships between halo and galaxy masses independently for the two classes of galaxies. When making halo occupation models split by colour, a convenient assumption is that passive and star-forming galaxies of the same stellar mass live in haloes of the same dark matter mass (e.g. Skibba \& Sheth 2009). This is also an inherent assumption of most galaxy group finders when the total stellar mass of a group is dominated by its central galaxy (Campbell et al. 2015). There is no reason a priori that this should be true, and the results of Tinker et al. (2013) show significant differences between the haloes of red and blue galaxies, especially at high stellar mass (see also More et al. 2011; Mandelbaum et al. 2016; Zu \& Mandelbaum 2016). Massive quiescent galaxies preferentially occupy more massive haloes than star-forming galaxies of the same mass at $z=0.3$.

To create this mock, we use the $z=0.3$ output of the MultiDark Planck-2 simulation (MDPL2; Prada et al. 2012), which is publicly available for download. ${ }^{2}$ Subhaloes are discarded and only host haloes are used. Host haloes are populated using the SHMRs for passive and active galaxies, including central and satellite populations. The cosmology assumed in Tinker et al. (2013) has $\Omega_{m}=0.27$, while MDPL2 has $\Omega_{m}=0.306$. To correct for this, we increase the halo mass scales in the SHMR fitting functions by a factor of $0.306 / 0.27=1.13$, which mostly corrects for the change in cosmology. As shown in Leauthaud et al. (2012), the COSMOS stellar masses are roughly 0.2 dex larger than the KCORRECT stellar masses used in the VAGC, so we shift all stellar masses down by 0.2 dex to put them on the same scale as the other mocks.

The results are shown in the bottom panel of Fig. 7. Because passive galaxies live in more massive haloes than star-forming galaxies of the same stellar mass, the environments of passive primary galaxies differ from that of star-forming primary galaxies. At $R_{\mathrm{p}}<3 \mathrm{Mpc}$, $f_{\mathrm{Q}}$ around passive primaries shows an increase over star-forming primaries, increasing to around $\sim 4$ per cent at $R_{\mathrm{p}}=1 \mathrm{Mpc}$.

\section{DISCUSSION}

\subsection{Comparison to previous work: theoretical}

We have shown that a conditional abundance matching model in which galaxy $D_{\mathrm{n}} 4000$ is connected to halo age can indeed produce strong galactic conformity out to $\sim 10 \mathrm{Mpc}$. In fact, most standard age-matching models that produce a strong signal at small scales $(\sim 2 \mathrm{Mpc})$ produce a signal at large scales. These results are in agreement with the previous results in Hearin et al. (2015). However, the amplitude of the conformity signal does depend on how age is defined. Models in which halo age is defined using peak halo mass rather than current halo mass - a process which limits the impact of tidal encounters on halo age - produces a much smaller conformity signal at all scales.

Bray et al. (2016) analyse galaxies in the Illustris cosmological hydrodynamic simulation in order to determine the conformity from that model of galaxy formation. Bray et al. (2016) find that the conformity signal in galaxies is roughly the same as the conformity signal in haloes (after dividing the halo population up into old and young subsets). The amplitude of the conformity, once restricted to primary and secondary galaxies both being central, is consistent with what we find in our standard age-matching models. Our

\footnotetext{
${ }^{2}$ https://www.cosmosim.org/cms/simulations/mdpl2/
}

$a_{\mathrm{c}}\left(M_{\text {peak }}\right)$ model yields a smaller conformity signal than found in Illustris, thus we conclude that tidal encounters have an immediate impact on star formation in galaxies in Illustris.

We demonstrated that backsplash galaxies can produce a conformity signal of a few percent at $R_{\mathrm{p}} \lesssim 3 \mathrm{Mpc}$. This is in contrast to Hearin et al. (2015), who analysed a backsplash model based on the results of Wetzel et al. (2014). They found no statistical evidence for conformity produced by such models. In this paper, we have used a simulation with four times the volume, increasing the statistical precision of the model and revealing the conformity signal seen in Fig. 7. Additionally, we have implemented the K13 isolation criterion to fully incorporate any observational effects.

\subsection{Comparison to previous work: observational}

For large-scale (a.k.a. 'two-halo') conformity, there is a surprising dearth of measurements for the local universe. The K13 measurements are specifically about sSFRs of secondary galaxies around primary galaxies. This work represents the first measurement of conformity using $f_{\mathrm{Q}}^{\text {cen }}$ as the statistic of interest. After correcting for contamination in the isolation criterion of $\mathrm{K} 13$, and restricting the secondary galaxies to be centrals of the same stellar mass range as the primaries, the conformity signal in SSFR is much closer to consistent with the $f_{\mathrm{Q}}^{\mathrm{cen}}$ results here.

Recently, Sin, Lilly \& Henriques (2017) also undertook a detailed re-examination of the K13 conformity measurement. They also found significant contamination of the sample of isolated galaxies by misclassified satellite galaxies. Additionally, they found that another source of conformity at detected at large scales can be produced by large groups with many satellites in proximity to one another, rather than correlated formation histories of low-mass central galaxies. The combination of our analysis and that of Sin et al. (2017) indicate that that the true level of large-scale conformity in SSFR values is small at best.

Hatfield \& Jarvis (2016) measured the angular clustering of photometrically selected galaxies. At $z \sim 1$, they cross-correlated highand low-mass galaxies, breaking both samples into passive and star-forming objects. They found that the amplitude of the crosscorrelation function of high-mass with low-mass passive galaxies is higher than high-mass galaxies crossed with low-mass star-forming galaxies. This is consistent with the effects of assembly bias, but it is difficult to disentangle the degeneracies in clustering amplitude between assembly bias, satellite fractions of red and blue galaxies, and the fact that red and blue galaxies of the same mass may occupy different mass haloes.

Berti et al. (2016) also use PRIMUS data to probe conformity in the redshift range $0.2<z<1$. In Berti et al. (2016), conformity is quantified by finding isolated 'central' galaxies using the K13 isolation criterion, and then measuring $f_{\mathrm{Q}}$ around passive and starforming primaries. At $R_{\mathrm{p}}<2 \mathrm{Mpc}$, they find that $f_{\mathrm{Q}}$ around passive primaries is between $1-4$ percent higher than around star-forming primaries. The signal detected is statistically robust, but is consistent with the amount of conformity seen in Section 3.3, either from backsplash galaxies or different halo occupation for star-forming and passive galaxies. Berti et al. (2016) find that $f_{\mathrm{Q}}$ around the two samples is consistent at $R>2 \mathrm{Mpc}$, which is inconsistent with the predictions of standard age matching; in Fig. 2, standard agematching models show a signal out to $10 \mathrm{Mpc}$ and beyond. The Berti et al. (2016) results are consistent with the expected age-matching signal from the $a_{\mathrm{c}}\left(M_{\text {peak }}\right)$ model as well as a model in which recent halo growth is used to define halo 'age'. But using conformity alone, it is impossible to distinguish the assembly bias effect in this 
model from the effects of splashback galaxies and differential halo occupation.

\subsection{One- and two-halo conformities}

Although the observational picture of large-scale conformity is murky at best, there is broad consensus that small-scale conformity - in which satellites within a dark matter halo are more likely to be passive if the central galaxy within that halo is passive - exists in the galaxy distribution. A number of authors, using different methods, have confirmed the original measurement of Weinmann et al. (2006b) (Phillips et al. 2014; Knobel et al. 2015; Kawinwanichakijet al. 2016; Berti et al. 2016).

One explanation for small-scale conformity is indelibly tied to halo assembly bias; older haloes are more likely to have subhaloes that are older - i.e. they were accreted longer ago - than younger haloes that are growing more rapidly. Older subhaloes are more likely to be quenched of their star formation (Weinmann et al. 2010; Wetzel et al. 2013), thus a correlation between host halo age and satellite galaxy colours fits naturally in this model. However, in order to produce conformity, the central galaxies in older haloes must be more likely to be quenched as well; i.e. two-halo conformity must exist because older haloes will be clustered with one another. How is it possible to achieve one-halo conformity without such conformity existing at larger scales?

Kauffmann (2015) proposes that active galactic nucleus heating - a mechanism widely invoked to quench star formation in central galaxies - can heat halo gas, causing increased efficiency of quenching nearby galaxies through ram pressure or reduced gas accretion. This mechanism is proposed to explain the K13 conformity signal that reached out to large scales, but should also apply to scales within the virial radius.

One caveat on the detection of small-scale conformity is that the halo masses are inferred or assumed, and biases may exist. In the group catalogue approach of Weinmann et al. (2006b), halo masses are assigned using the total stellar mass of the group. For $M_{\mathrm{h}} \lesssim 10^{13}$ $\mathrm{M}_{\odot}$, the total mass is dominated by the central galaxy. Thus, the group finder assumes near one-to-one correlation between central galaxy stellar and host halo masses, regardless of whether the galaxy is star forming or quiescent. As discussed in Section 3.3, this assumption that star-forming and quiescent galaxies of the same $M_{*}$ live in haloes of the same dark matter mass is not supported by studies constraining the halo occupation of these two types of galaxies independently. Thus, halo masses assigned in group catalogues may be biased when split on central galaxy colour: the haloes around quiescent central galaxies would be underestimated, while those around star-forming central galaxies would be overestimated. This could impart some conformity between the properties of satellite galaxies and their host centrals when using group-catalogue halo masses, but the impact of this bias has not been quantified. We leave more thorough investigation of this effect to another paper in this series. Within semi-analytic galaxy formation models, Wang \& White (2012) found that massive, isolated, quiescent galaxies have more red satellites than their star-forming counterparts for this very reason: they live in more massive haloes.

\subsection{Halo formation and central galaxy quenching}

Is not the scenario described above - in which star-forming and passive galaxies of the same stellar mass occupying different haloes - itself a manifestation of galaxy assembly bias? Possibly, but a correlation between galaxy and halo assemblies is not required to create this scenario. If the process by which galaxies stop forming stars is entirely stochastic at fixed halo mass, but the efficiency of this process increases monotonically with $M_{\mathrm{h}}$ - or a galaxy property the correlates with $M_{\mathrm{h}}$, like $M_{*}$ - you naturally end up with passive galaxies living in higher mass haloes at fixed stellar mass: the haloes of both passive and star-forming galaxies continue to grow, but only the star-forming galaxies increase their mass. Thus, at fixed halo mass, the passive galaxies are smaller than the star-forming ones (which translates into higher halo masses for passive galaxies at fixed halo mass).

However, in order to wipe out any correlations with halo assembly history in the present-day universe, the efficiency of the quenching mechanism can only be correlated with the eventual $z=0$ halo mass, and not the mass of the halo at the time at which the quenching occurs. For example, if quenching occurs at some threshold in halo mass, early-forming haloes will reach that threshold earlier than later-forming haloes. The same correlation would exist if the threshold for quenching was in galaxy stellar mass, under the assumption that early-forming haloes would have more massive galaxies even if all haloes converted the same fraction of accreted baryons into stars (see e.g. Tinker 2016). Even a model in which quenching is not due to crossing a threshold but rather due to a process which imparts a quenching probability that varies continuously - and probably monotonically - with halo mass and redshift would contain some imprint of halo formation history because the early-forming haloes would have a higher quenching probability at a given redshift. The degree to which this is represented in the spatial distribution of quenched galaxies will in large degree be reflective of how steep the quenching probability is with $M_{\mathrm{h}}$.

From Paper I and this paper, we have observational evidence that the correlation between the quenching mechanism and halo formation history is weak but non-zero for massive galaxies (shown in Paper I) and close to negligible for lower mass galaxies (shown in both papers). This supports a model in which quenching of galaxies is a stochastic process, where the probability of going through the quenching process is a weak function of mass at low masses and strong function of mass - closer to a threshold - for higher masses. The evidence presented here does not indicate which mass is most important: whether the quenching probability is determined by $M_{\mathrm{h}}$ or $M_{*}$. Because of the tight correlation between the two, a quenching threshold in one property would induce a correlation between quenching probability and the other quantity. Tinker (2016) proposes using the scatter in the relationship between stellar and halo masses to distinguish between these two scenarios. Gu, Conroy \& Behroozi (2016) have demonstrated that star formation, rather than merging, is the dominant contributor to this scatter for all but the most massive galaxies and haloes.

With the measurements put forward in this paper and Paper I, in combination with other measurements, there should now be enough data to constrain the relationship between central galaxy quenching and halo mass and formation history. This combination includes the scatter in relationship between halo and stellar masses, the fraction of central galaxies that are quenched and how this quantity depends on $M_{*}$ and redshift, constraints on the SHMRs of star-forming and quiescent galaxies, and the existence of small-scale galactic conformity. These data present a wealth of information to constrain how the quenching probability depends on $M_{\mathrm{h}}$ and redshift. Armed with this knowledge, we will take a major step forward in understanding which physical mechanisms are most important for quenching, which are secondary correlations without causation, and which are uncorrelated with the process that stops stars from being formed in central galaxies. 


\section{CONCLUSIONS}

We have measured the galactic conformity signal around central galaxies using group catalogues to isolate 'primary' central galaxies. The quantity we measure is the quenched fraction of central galaxies around primary central galaxies that have been divided up by their star formation activity - i.e. a quenched sample and a starforming sample. We then compare these measurements to different theoretical models that vary in how galaxy stellar age is correlated (or not) with halo age, using various definitions of halo age. We focus on scales larger than $1 \mathrm{Mpc}$ in order to isolate effects between pairs of distinct haloes, rather than galaxies that share the same host halo. We find the following:

(i) In SDSS DR7 data, there is little no to statistical evidence of any difference in the $f_{\mathrm{Q}}$ of central galaxies around star-forming and quiescent centrals.

(ii) If galaxy quenching were correlated with halo age - using halo age definitions such as $z_{1 / 2}, a_{\mathrm{c}}\left(M_{\mathrm{h}}\right)$, and halo growth since $z=0.8-$ there would be a significantly detectable $(\gtrsim 3 \sigma)$ signal of galactic conformity. We do not detect this.

(iii) Other definitions of halo age, such as $a_{\mathrm{c}}\left(M_{\text {peak }}\right)$ and halo growth over shorter redshift intervals like $\Delta z=0.1$, would not yield a detectable conformity signal in the data.

(iv) The strong conformity signal in galaxy star formation rates seen in K13 is almost entirely eliminated by removing a small number of satellite galaxies that are not excluded in the K13 isolation criterion.

(v) At $1<R<3 \mathrm{Mpc}$, small conformity signals in $f_{\mathrm{Q}}$ can be created by means other than galaxy assembly bias.

\section{ACKNOWLEDGEMENTS}

The authors wish to thank Michael Blanton and Risa Wechsler for many useful discussions. ARW was supported by a Caltech-Carnegie Fellowship, in part through the Moore Center for Theoretical Cosmology and Physics at Caltech. The authors thank Matthew R. Becker for providing the Chinchilla simulation used in this work. The Chinchilla simulation and related analysis were performed using computational resources at SLAC. We thank the SLAC computational team for their consistent support. The authors acknowledge the Gauss Centre for Supercomputing e.V. (www.gauss-centre.eu) and the Partnership for Advanced Supercomputing in Europe (www.prace-ri.eu) for funding the MultiDark simulation project by providing computing time on the GCS Supercomputer SuperMUC at Leibniz Supercomputing Centre (www.lrz.de). The Bolshoi simulations have been performed within the Bolshoi project of the University of California HighPerformance AstroComputing Center and were run at the NASA Ames Research Center.

\section{REFERENCES}

Abazajian K. N. et al., 2009, ApJS, 182, 543

Behroozi P. S., Wechsler R. H., Wu H.-Y., Busha M. T., Klypin A. A., Primack J. R., 2013, ApJ, 763, 18

Behroozi P. S., Wechsler R. H., Wu H.-Y., 2013, ApJ, 762, 109

Berti A. M., Coil A. L., Behroozi P. S., Eisenstein D. J., Bray A. D., Cool R. J., Moustakas J., 2016, ApJ, 834, 87
Blanton M. R., Moustakas J., 2009, ARA\&A, 47, 159

Blanton M. R., Roweis S., 2007, AJ, 133, 734

Blanton M. R. et al., 2005, AJ, 129, 2562

Bray A. D. et al., 2016, MNRAS, 455, 185

Brinchmann J., Charlot S., White S. D. M., Tremonti C., Kauffmann G., Heckman T., Brinkmann J., 2004, MNRAS, 351, 1151

Campbell D., van den Bosch F. C., Hearin A., Padmanabhan N., Berlind A., Mo H. J., Tinker J., Yang X., 2015, MNRAS, 452, 444

Gao L., White S. D. M., 2007, MNRAS, 377, L5

Gu M., Conroy C., Behroozi P., 2016, ApJ, 833, 2

Hahn C., Tinker J. L., Wetzel A. R., 2016, ApJ, 841, 6

Hatfield P. W., Jarvis M. J., 2016, MNRAS, 472, 3570

Hearin A. P., Watson D. F., 2013, MNRAS, 435, 1313

Hearin A. P., Watson D. F., van den Bosch F. C., 2015, MNRAS, 452, 1958

Kauffmann G., 2015, MNRAS, 454, 1840

Kauffmann G., Li C., Zhang W., Weinmann S., 2013, MNRAS, 430, 1447

Kawinwanichakij L. et al., 2016, ApJ, 817, 9

Klypin A., Yepes G., Gottlöber S., Prada F., Heß S., 2016, MNRAS, 457, 4340

Knobel C., Lilly S. J., Woo J., Kovač K., 2015, ApJ, 800, 24

Leauthaud A. et al., 2012, ApJ, 744, 159

Li Y., Mo H. J., Gao L., 2008, MNRAS, 389, 1419

Maller A. H., Berlind A. A., Blanton M. R., Hogg D. W., 2009, ApJ, 691, 394

Mandelbaum R., Wang W., Zu Y., White S., Henriques B., More S., 2016, MNRAS, 457, 3200

Masters K. L. et al., 2010, MNRAS, 404, 792

More S., van den Bosch F. C., Cacciato M., Skibba R., Mo H. J., Yang X., 2011, MNRAS, 410, 210

Peng Y., Maiolino R., Cochrane R., 2015, Nature, 521, 192

Phillips J. I., Wheeler C., Boylan-Kolchin M., Bullock J. S., Cooper M. C., Tollerud E. J., 2014, MNRAS, 437, 1930

Prada F., Klypin A. A., Cuesta A. J., Betancort-Rijo J. E., Primack J., 2012, MNRAS, 423, 3018

Sin L. P. T., Lilly S. J., Henriques B. M. B., 2017, MNRAS, 471, 1192

Skibba R. A., Sheth R. K., 2009, MNRAS, 392, 1080

Tinker J. L., 2016, MNRAS, 467, 3533

Tinker J., Wetzel A., Conroy C., 2011, MNRAS, preprint (arXiv:1107.5046)

Tinker J. L., Leauthaud A., Bundy K., George M. R., Behroozi P., Massey R., Rhodes J., Wechsler R. H., 2013, ApJ, 778, 93

Tinker J., Wetzel A., Conroy C., Mao Y.-Y., 2016, MNRAS, 472, 2504

Wang W., White S. D. M., 2012, MNRAS, 424, 2574

Wechsler R. H., Bullock J. S., Primack J. R., Kravtsov A. V., Dekel A., 2002, ApJ, 568, 52

Wechsler R. H., Zentner A. R., Bullock J. S., Kravtsov A. V., Allgood B., 2006, ApJ, 652, 71

Weinmann S. M., van den Bosch F. C., Yang X., Mo H. J., 2006a, MNRAS, 366,2

Weinmann S. M., van den Bosch F. C., Yang X., Mo H. J., 2006b, MNRAS, 366,2

Weinmann S. M., Kauffmann G., von der Linden A., De Lucia G., 2010, MNRAS, 406, 2249

Wetzel A. R., Tinker J. L., Conroy C., van den Bosch F. C., 2013, MNRAS, 432, 336

Wetzel A. R., Tinker J. L., Conroy C., Bosch F. C. v. d., 2014, MNRAS, 439, 2687

Zu Y., Mandelbaum R., 2016, MNRAS, 457, 4360

This paper has been typeset from a $\mathrm{T}_{\mathrm{E}} \mathrm{X} / \mathrm{L} \mathrm{T} \mathrm{E} \mathrm{X}$ file prepared by the author. 